\title{
Comparison of the Metabolic Process of Bone Mineral Density of Females during the Life Cycle using Allometric Scaling Techniques
}

\author{
M Bonis ${ }^{1 *}$, M Loftin $^{2}$ and M Sothern ${ }^{3}$ \\ ${ }^{1}$ University of New Orleans, USA \\ ${ }^{2}$ University of Mississippi, USA \\ ${ }^{3}$ Louisiana State University Health Science Center, New Orleans, USA
}

*Corresponding author: M P Bonis, Assistant Professor New Orleans, Human Performance and Health Promotion Program, School of Education, 2000 Lakeshore Drive ED 348K, New Orleans, Louisiana 70148, USA

To Cite This Article: M Bonis, M Loftin, M Sothern, Comparison of the Metabolic Process of Bone Mineral Density of Females during the Life Cycle using Allometric Scaling Techniques. 2020 - 8(6). AJBSR.MS.ID.001323. DOI: 10.34297/AJBSR.2020.08.001323.

Received: 淎 April 28, 2020; Published: 眥 May 07, 2020

\begin{abstract}
Purpose: The purpose of the study was to determine the best body composition predictor for

BMD from 4 different age groups of women, compare the groups' bone mineral density (BMD), and examine these groups using allometric scaling techniques.
\end{abstract}

Methods: 163 female subjects (mean age+SD = 34.4+22.2 years) were divided into 4 age group

categories: adolescent, young adult, middle-age adult, and the elderly. Body composition measurements using dual-energy x-ray absorptiometry (DXA) were conducted. Height and weight measurements were also collected.

Results: Statistical analysis revealed that lean tissue was the best body composition predictor.

Univariate ANOVA analyses revealed significant differences among the age groups for BMD, F $(3,159)=33.23, \mathrm{p}<0.05, \mathrm{\eta}^{2}=0.385$ and for LT, $\mathrm{F}(3,159)=11.55, \mathrm{p}<0.05, \mathrm{y}^{2}=0.179$. Post hoc tests indicated that the adolescent and elderly groups' BMD were significantly different than the young and middle-age groups. The adolescent group's LT was significantly different than the young and middle-age groups, and the elderly group was significantly different than the young age group. The allometric value for the total study was BMD $=.237 \mathrm{LT} .^{431}$ with the coefficient range being $0.196-0.268$, the adolescent value was BMD $=.277 \mathrm{LT} .379$, the young adult value was $\mathrm{BMD}=.402 \mathrm{LT}^{301}$, the middle age adult value was $\mathrm{BMD}=.378$ $\mathrm{LT}^{313}$, and the elderly group value was BMD $=.356 \mathrm{LT}^{312}$.

Conclusion: Allometric scaling techniques provide new ways to examine BMD, and may provide deeper insights into understanding the complex association between BMD and body composition. Further investigation between BMD and allometric scaling of body composition components should be conducted in larger samples, mixed ethnic, and gender groups.

Keywords: Allometric scaling, Bone mineral density, Body composition, Lean tissue

\section{Introduction}

Genetics is the major determinant of bone mineral density (BMD) in adults, but body composition and physical activity are major factors in attaining peak BMD during adolescence [1]. The pre-pubertal human skeleton is sensitive to the mechanical stimulation by physical activity. High volumes of weight-bearing physical activity place more mechanical strain on the skeleton and stimulate metabolic acidosis which promotes bone remodeling
$[2,3]$. Therefore, to obtain the maximal peak bone mass potentially achievable, physical activity should start at pre-pubertal ages and should be maintained throughout pubertal development.

Physical activity that stimulates BMD also stimulates lean tissue growth [4]. Starting physical activity prior to the pubertal growth spurt stimulates both bone and skeletal muscle hypertrophy to a greater degree than observed with normal growth in non- 
physically active children. Participation in weight-bearing physical activities are strongly recommended to increase the peak bone mass. Further, research in 2012 by Vicente-Rodriguez found the increase in lean mass is the most important predictor for bone mineral mass accrual during pre-pubertal growth throughout the population. Since skeletal muscle is the primary component of lean mass, participation in pre-pubertal physical activity could not only have a direct osteogenic effect, but also an indirect effect by increasing muscle mass [5].

Given these findings, the current study sought to determine body composition components that influence BMD in females. Females have a number of unique physiological issues compared to their male counterparts. Young female athletes who are driven to excel athletically and desire to maintain a certain body image are at risk for developing three distinct, interrelated disorders, disordered eating, amenorrhea, and osteoporosis. Together these three disorders are commonly referred to as the female athlete triad syndrome [6,7]. Disordered eating is a condition that ranges from improper nutritional intake to eating disorders, such as anorexia nervosa and bulimia, and is related to amenorrhea and lower BMD [8,9]. Amenorrhea is associated with higher injury rates, such as stress fractures, and lower BMD in female athletes $[10,11]$. These two disorders are interrelated and, if left untreated, place the athlete at greater risk of the third and final disorder of the syndrome, osteoporosis [12].

Body mass (Wgt), fat (BF), lean tissue (LT), and body mass index (BMI) are body composition components that are used in studying BMD. The Body Mass Index (BMI) is also used in body composition studies because it incorporates bodyweight and height into one term and provides a measure of size. It is defined as total body weight (in kilograms) divided by the height (in meters) squared. BMI can be separated into its components, the LTI, BFI, and the BI, and utilized in lieu of the BMI in certain situations to provide greater insight into the associations among body composition components and BMD [13]. In a previous study [14], fat (BFI) and fat-free (LTI) indices were used in a multiple regression analysis to determine what body size and body composition variables were most highly associated with physical activity. [Note: LTI is defined as total lean tissue (in kilograms) divided by the height squared (in meters). BFI is defined as total body fat (in kilograms) divided by the height squared (in meters); and BI is defined as total bone mineral content (in kilograms) divided by the height squared (in meters).]

Physiological processes, like metabolism, are not linear relationships; however, researchers use parametric linear methods to analyze, examine, and describe them. Occasionally researchers convert variable data into logarithmic values to yield more reliable conclusions about these processes by fitting non-linear data into linear models.

There are other ways to examine metabolic processes, like BMD. The allometric scale uses parameters that allow comparison of physiological variables between individuals with different masses $[15,16]$. It is represented by means of a non-linear equation that indicates the behavior of a physiological variable $\mathrm{Y}$ in relation to the variable mass $\mathrm{X}(\mathrm{Y}=\mathrm{aXb})$, where a is the allometric coefficient (a constant that is characteristic for the organism) and $b$ is the allometric or scaling exponent. This exponential function can be transformed into a linear function: $\log y=\log a+b \log x$ [17] which also has a linear regression format. Previous research has employed allometric scaling to examine V02submax and VO2peak values from different sizes and ages of humans [18,19], but BMD has not been examined using allometric scaling of body mass and body mass components.

The purpose of this study was to investigatethe relationships of BMD and to:

1. Determine the best body composition predictor variable for BMD in the study.

2. Determine if any significant BMD differences exist among the four different age groups in the study.

3. Convert the linear relationships between BMD and the best predictor variable of the study into allometric equations to examine additional information about the BMD process.

\section{Methods}

\section{Participants}

The participants of the study consisted of 163 females (mean age+SD=34.4+22.25 years) divided into 4 different age categories: the adolescent group consisting of 62 subjects (mean age $+\mathrm{SD}=12.6+1.2$ years), the young adult group consisting of 35 subjects (mean age $+\mathrm{SD}=27.2+7.1$ years), the middle-age group consisting of 36 subjects (mean age $+\mathrm{SD}=49.7+4.8$ years), and the elderly group consisting of 30 subjects (mean age $+\mathrm{SD}=69.4+5.8$ years) (See Table 1). Data from the current study was secondary data taken from four previously IRB-approved convenience studies conducted in the exercise physiology lab at the University of New Orleans more than 10 years ago. All of the data from the four studies were collected by the author of the current study using the same equipment.

\begin{tabular}{|c|c|c|c|c|c|c|c|}
\hline \multicolumn{1}{|l|}{ Table 1: Physical Characteristics } \\
\hline Group & $\mathbf{N}$ & Age-yrs & Hgt-m & Wgt-kg & Lean-kg & Fat-kg & BMI \\
\hline Total & 163 & $34.4+22.2$ & $1.60+0.10$ & $62.4+16.6$ & $39.3+7.21$ & $20.0+11.0$ & $24.1+5.4$ \\
\hline Adoles. & 62 & $12.6+1.2$ & $1.54+0.08$ & $54.7+18.2$ & $36.0+7.31$ & $15.5+11.2$ & $22.5+6.1$ \\
\hline
\end{tabular}




\begin{tabular}{|c|l|l|l|l|l|r|r|}
\hline Young & 35 & $27.2+7.1$ & $1.69+0.11$ & $65.7+11.9$ & $43.9+7.94$ & $19.1+7.7$ & $23.3+2.8$ \\
\hline Middle & 36 & $49.7+4.8$ & $1.63+0.07$ & $68.2+15.7$ & $40.8+5.05$ & $23.9+11.0$ & $25.8+5.5$ \\
\hline Elderly & 30 & $69.4+5.8$ & $1.59+0.06$ & $67.7+12.7$ & $38.8+4.64$ & $25.7+9.7$ & $26.6+4.6$ \\
\hline
\end{tabular}

In the adolescent study prior to participation the investigator explained the experimental protocol to the subjects and their parents. The parents received a Parental Permission/Informed Consent form and the subjects were given a Minor Subject's Assent Form to read and sign before any research began. They were told the purpose of the study, that their participation was completely voluntary, and that they had the right to refuse to participate or withdraw from the study at any time. The subjects were a convenience sample of females recruited from middle schools and high schools (grades 6, 7, 8 \& 9) from the New Orleans area. In the remaining three studies the subjects were told the purpose of the study, that their participation was completely voluntary, and that they had the right to refuse to participate or withdraw from the study at any time. The subjects were a convenience sample of females recruited from the greater New Orleans area. All four studies were approved by the Institutional Review Board (IRB) of the University of New Orleans.

The current study is of particular interest to females who can undergo unique BMD issues during the growth spurt that are related to disorders such as amenorrhea and the female athlete triad syndrome $[6,7,12,20,21]$ and to post-menopausal women who are at risk for osteopenia and osteoporosis issues [1- 5]. The researchers included only female volunteers in the BMD study to exclude any gender-confounding factors.

\section{General Experimental Procedure}

Body composition measurements were conducted on the subjects using dual-energy x-ray absorptiometry (DXA). The subjects were also weighed andmeasured for height.

\section{Procedures}

All measurements were performed at the University of New Orleans according to standard procedures as specified by the equipment manufacturers. Body composition was obtained from whole-body scans using dual-energy x-ray absorptiometry (DXA). A Lunar DPX-L densitometer was used to perform full body scans. Subjects were positioned according to the standard protocols for whole body scans. Analysis was determined using the manufacturer's automated algorithms. Subjects were scanned in light-weight clothing after removing all jewelry. The variables measured included fat-mass, fat-free mass, bone mineral content, bone calcium, and bone mineral density. The DXA was calibrated before each scan. Standing height was measured in centimeters using a fixed Schorr stadiometer, and body weight was measured in kilograms using the Seca Model 780 scale calibrated with a standard weight.

\section{Analysis}

IBM SPSS Statistics Version 24 for Windows was used for statistical analysis. Pearson Product-moment correlations and linear regressions were used to examine the relationships among BMD and body mass (Wgt), lean tissue (LT), fat (BF), body mass index (BMI), and lean tissue index (LTI).

Simple linear regression analysis was used to predict the dependent criterion variable, BMD, using the previously noted body composition components as independent predictor variables. Analysis of variance (ANOVA) was used to determine the significance of the regression models. Mahalanobis distance and chi square critical values were determined to identify data outliers, and residual plots were used to evaluate univariate normality. Univariate analysis of variance (ANOVA) was used to compare the BMD and the best predictor variable between the four age groups in the study. The best predictor variable was used to determine the BMD allometric relationships. Allometric scaling of the metabolic process of BMD was applied using the following procedure:

a. After the strongest associated predictor variable is selected, substitute it into the allometric logarithmic formula as the independent variable.

b. If the simple regression analysis results reveal that the predictor variable is significant then the unstandardized coefficient and constant can be used and the anti-log results will yield an allometric coefficient that describes the relative relationship of the sample to BMD and the allometric exponent will describe the metabolic growth that is characteristic of the sample.

c. If more than one predictor variable is significant in reviewing the various simple linear regression analyses, select the predictor with the best fit, or greatest coefficient of determination, $\mathrm{R}^{2}$.

d. Holding the sample's allometric exponent constant and substituting the individual subjects' LT value will yield the individual subject's characteristic allometric coefficient in regard to BMD [16]. Using these calculated coefficient values hypothetical BMD values can be calculated and compared to the actual measured BMD values. Pearson Product correlation analysis between the current study $\mathrm{BMD}_{\text {measured }}$ vs. the current

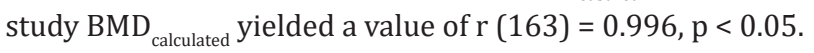

This procedure is predicated on the fact that the initial simple regression analysis must indicate that there is a significant relationship between the predictor variable and BMD, the outcome 
variable. If the relationship is not significant then the BMD process for that sample cannot be validly expressed using that predictor variable.

\section{Limitations}

Thirty-eight percent (38.0\%) of the subjects were adolescents, while $21.5 \%$ were from the young adult group, and $22.1 \%$ and $18.4 \%$ were from the middle and elderly age groups, respectively. There is a concern that the allometric scaled predictor value calculated for the total sample may be positively skewed by the adolescent group.

\section{Results}

Univariate ANOVA analyses were conducted to compare the bone mineral density values and lean tissue values among the four age groups. Analyses revealed significant differences among the age groups for BMD, $\mathrm{F}(3,159)=33.23, \mathrm{p}<0.05, \mathrm{n}^{2}=0.385$ and for $\mathrm{LT}$,
$\mathrm{F}(3,159)=11.55, \mathrm{p}<0.05, \mathrm{y}^{2}=0.179$. Post Hoc analyses indicated the BMD mean values of the adolescent group $\left(1.07+0.10 \mathrm{gm} / \mathrm{mm}^{2}\right)$ and the elderly group $\left(1.12+0.08 \mathrm{gm} / \mathrm{mm}^{2}\right)$ were both significantly different from the young adult group $\left(1.25+0.09 \mathrm{gm} / \mathrm{mm}^{2}\right)$ and the middle age adult group $\left(1.21+0.09 \mathrm{gm} / \mathrm{mm}^{2}\right)$. Similarly, the LT mean values of the adolescent group $(35.97+7.31 \mathrm{~kg})$ and the elderly group $(38.82+4.64 \mathrm{~kg})$ were both significantly different than the LT mean values of the young adult group (43.90+7.94 kg), and theadolescent group was also significantly different from the middle-age adult group $(40.76+5.05 \mathrm{~kg})$.

Pearson product correlations, simple linear regressions, and coefficients of determination were run to determine the strongest body composition predictor variable for bone mineral density. Lean tissue (LT) was determined to be the strongest significant predictor variable for BMD $[\rho(163)=0.736]$ and the best fit, accounting for $54.2 \%$ of the relationship. See Tables 2 and 3.

\begin{tabular}{|c|c|c|c|c|c|c|}
\hline Group & $\mathbf{N}$ & Weight & Lean Tissue & Fat & BMI & LTI \\
\hline Total & 163 & $.590^{* *}$ & $.736^{* *}$ & $.389 * *$ & $.393^{* *}$ & $.477^{* *}$ \\
\hline Adolescents & 62 & $.685^{* *}$ & $.743^{* *}$ & $.600^{* *}$ & $.641^{* *}$ & $.664^{* *}$ \\
\hline Young & 35 & $.541^{* *}$ & $.761^{* *}$ & 0.008 & 0.075 & $.481^{* *}$ \\
\hline Middle & 36 & 0.511 & $.550 * *$ & 0.472 & 0.469 & $.469^{* *}$ \\
\hline Elderly & 30 & 0.302 & $.467^{* *}$ & 0.119 & 0.16 & $.246^{* *}$ \\
\hline
\end{tabular}

* Significant at the 0.01 level

Table 3: Predictor Variables R2 Values for BMD.

\begin{tabular}{|c|c|c|c|c|c|}
\hline Group & N & LT (kg) & Weight (kg) & Fat (kg) & BMI \\
\hline Total & 163 & 0.542 & 0.355 & 0.16 & 0.349 \\
\hline Adolescents & 62 & 0.551 & 0.461 & 0 & 0.411 \\
\hline Young & 35 & 0.579 & 0.294 & 0.44 \\
\hline Middle & 36 & 0.283 & 0.221 & 0.255 & 0.231 \\
\hline Elderly & 30 & 0.222 & 0.093 & 0.014 & 0.026 \\
\hline
\end{tabular}

Residual scatterplots were generated to evaluate univariate normality. No transformations of variables were required. Multicollinearity was also confirmed. All independent variables had tolerance values greater than 0.2 and VIF values less than 5.0.

ANOVA indicated a significant relationship, $F(1,161)=194.4, p<$ 0.05 , between BMD and LT, the best predictor variable. The simple linear regression model with the best fit was:

$$
\mathrm{BMD}=.671+0.012 \mathrm{LT}
$$

The Levene test of homogeneity of variances indicated that the variances for the BMD groups were homogeneous, $F(3,159)=1.29$, $\mathrm{p}>0.05$.

Simple linear regression was used to solve the allometric scale relationship $[15,22,17,24]$. The strongest body composition predictor variable, LT, was used as variable, $\mathrm{X}$, to describe the dependent physiological variable, BMD, or Y:

$$
\mathrm{Y}=\mathrm{a} \mathrm{X}^{\mathrm{b}}
$$

$\mathrm{BMD}=\mathrm{a}(\mathrm{LT})^{\mathrm{b}}$

Taking the logarithm of both sides of the relationship yields the logarithmic equation:

$\log B M D=\log \left[\mathrm{a}(\mathrm{LT})^{\mathrm{b}}\right]$

$\log B M D=\log a+b \log L T$

This resultant logarithmic equation has the same format as a simple linear regression. Data variables, LOG BMD and LOG LT, were inserted into a simple linear regression formula with LOG BMD being the dependent variable and Log LT the independent variable. ANOVA indicated a significant relationship, $F(1,161)=214.5, p<$ 0.05. The regression equation results were:

$\log$ BMD $=-.626+.431 \log$ LT 
Taking the inverse logarithm of the resultant equation yields the following:

$\log ^{-1}[\log B M D]=\log ^{-1}[-.626+.431 \log \mathrm{LT}]$

So:

$\log \mathrm{a}=-.626$

$\log -1(\log a)=\log -1(-.626)=.237$

$\mathrm{a}=.237=$ allometric coefficientmean

$\mathrm{b}=.431=$ allometric exponent

The resultant allometric BMD relationship for the study was:

$\mathrm{BMD}=.237 \mathrm{LT}^{431}$
The mean coefficient for the group was .237. Holding the allometric exponent constant at LT. $^{431}$ and substituting the individual values of BMD and LT. ${ }^{431}$, the individual coefficients for each of the subjects can be determined. The coefficient range for the sample was $.196-.268$, with .237 being the coefficient mean [16]. Multiplying the individual coefficient values times the individual $\mathrm{LT}^{431}$ values yielded $\mathrm{BMD}_{\text {calculated }}$ values. Pearson product correlation analysis between $\mathrm{BMD}_{\text {measured }}$ and $\mathrm{BMD}_{\text {calculated }}$ values yielded a correlation of:

$$
\mathrm{r}(163)=0.996, \mathrm{p}<0.05
$$

Using the same procedure for each of the study's age group data values yielded the followinggroup allometric equations (See Table 4):

\begin{tabular}{|c|c|c|c|c|}
\hline Table 4: BMD Allometric Scale Values \\
\hline Group & $\mathrm{N}$ & Age & BMD = A LTB & "A" Range \\
\hline Total & 163 & $34.4+22.2$ & BMD $=.237$ LT.431 & $.196-.268$ \\
\hline Adolescents & 62 & $12.6+1.2$ & BMD $=.277$ LT.379 & $.242-.318$ \\
\hline Young & 35 & $27.2+7.1$ & BMD $=.402$ LT.301 & $.362-.448$ \\
\hline Middle & 36 & $49.7+4.8$ & BMD $=.378$ LT.313 & $.329-.415$ \\
\hline Elderly & 30 & $69.4+5.8$ & BMD $=.356$ LT.312 & $.314-.405$ \\
\hline
\end{tabular}

Adolescent Group:

$\log B M D=-.558+.379 \log$ LT

BMD $=.277$ LT. $^{379}$ Coefficient Range $.242-.318$

Young Adult Group:

$\log$ BMD $=-.396+.301 \log$ LT

BMD $=.402$ LT $^{301}$ Coefficient Range $.362-.448$

Middle-aged Group:

$\log B M D=-.423+.313 \log$ LT

BMD $=.378$ LT. $^{313}$ Coefficient Range $.329-.415$
Elderly Group:

$\log B M D=-.449+\log \mathrm{LT}$

BMD $=.356$ LT $^{312}$ Coefficient Range $.314-.405$

While these individual allometric equations reflect the mathematical results of the age groups they do not provide a good method for comparing the groups. The individual age group equations can be normalized for better group comparisons. Normalization of the individual allometric equations can be converted by changing the allometric exponent for each age group to the total study allometric exponent and solving for the normalized equation. Doing this resulted in the following equations (See Table 5):

\begin{tabular}{|c|c|c|c|c|}
\hline \multicolumn{1}{|l|}{ Table 5: Normalized BMD Allometric Scale Values } \\
\hline Group & $\mathbf{N}$ & Age (yrs) & BMD = A (LT)B & "A" Range \\
\hline Total & 163 & $34.4+22.2$ & BMD $=.237$ LT.431 & $.196-.268$ \\
\hline Adolescents & 62 & $12.6+1.2$ & BMD $=.230$ LT.431 & $.197-.262$ \\
\hline Young & 35 & $27.2+7.1$ & BMD $=.246$ LT.431 & $.216-.268$ \\
\hline Middle & 36 & $49.7+4.8$ & BMD $=.243$ LT.431 & $.212-.267$ \\
\hline Elderly & 30 & $69.4+5.8$ & BMD $=.231$ LT.431 & $.196-.262$ \\
\hline
\end{tabular}

Adolescent Group:

$\mathrm{BMD}=.277 \mathrm{LT}^{.379}=\mathrm{x} \mathrm{LT} \cdot 431$

BMD = .230 LT.431 Coefficient Range .197 - .262

Young Adult Group:

$\mathrm{BMD}=.402 \mathrm{LT}^{.301}=\mathrm{x} \mathrm{LT} \cdot 431$
$\mathrm{BMD}=.246 \mathrm{LT}^{.431}$ Coefficient Range .216 - .268

Middle-aged Group:

BMD $=.378$ LT.313 = x LT.431

BMD = .243 LT $^{431}$ Coefficient Range $.212-.267$

Elderly Group: 


$$
\begin{aligned}
& \text { BMD }=.356 \mathrm{LT}^{312}=\mathrm{x} \mathrm{LT}^{.431} \\
& \text { BMD }=.231 \mathrm{LT}^{431} \text { Coefficient Range } .196-.262
\end{aligned}
$$

\section{Discussion}

The current research found that LT( $r=0.736)$ was the best body composition component associated with BMD in the study. The simple linear regression result was the equation:

$\mathrm{BMD}=.671+.012 \mathrm{LT}$

Univariate analysis indicated that the BMD for the young adult and middle-aged groups were similar and significantly greater than the adolescent and elderly groups, and the LT for the young and middle-aged groups were also significantly greater than the adolescent group.

Research by Huxley [22] recognized that even though physiological traits were strongly associated many of these associations were curvilinear, and transforming collected data to its $\log 10$ values made the statistical linear analyses stronger. The current research supports the research in the relationship between BMD and the best body composition predictor, LT. Pearson correlation results between Log BMD and Log LT reveal $r=.756$ while correlation results between BMD vs LT was $r=.736$; and the coefficient of determination between Log BMD and Log LT was $\mathrm{R}^{2}=$ .571 , while the coefficient of determination between BMD vs LT was $\mathrm{R}^{2}=.542$. Using logarithmic relationships Huxley and Tessier [17] developed a simple linear regression format to describe allometric scaling. The computed allometric relationship between BMD and LT for the current study population indicated that BMD is associated with lean tissue that developed at a power of 0.431 .

$\mathrm{BMD}=.230 \mathrm{LT}^{4} 431$

White and Gould [16], in interpreting the allometric general equations, stated the allometric coefficients denote relative magnitudes between corresponding data sets while holding the allometric exponent constant. The current study results indicated the range of coefficient values for the sample and the four age groups (See Tables $4 \& 5$ ). A review of the individual coefficients of the study indicated that the magnitude of the allometric coefficient increased with age until in the middle-aged group the allometric coefficient magnitude began to decrease in the subjects at approximately forty-five years of age. This resultant decrease in BMD in the allometric equation could possibly correspond to female menopause. Additional research would corroborate these findings.

Another interesting observation in the study was the possible application of Klieber's Lawwhich states that the physics and geometry of trait to body size in animal circulatory and metabolic processes should approximate the .75 power [15,22,17,23,24]. The standardized beta coefficient from the current regression analysis was $(\beta=0.756)$. This calculated relationship approximated Klieber's Law. From a statistical perspective the beta coefficient is the correlation between the target and predictor variables, but from an allometric scaling standpoint it is the exponential relationship between bone mineral density and the metabolic growth rate of lean tissue. It would be interesting to see in future allometric studies if the beta coefficient would also generate a value approximating Klieber's Law.

From the current study BMD $=\mathrm{LT}^{7} \cdot 76$

Substantial research has investigated the relationships among BMD, body composition components, and physical activity. The current research found LT was the body composition component most associated with BMD ( $\mathrm{r}=.736)$ for the subjects (mean age+SD $=34.4+22.2 \mathrm{yrs}$ ). It also supported previous research by VicenteRodriguez that concluded LT is the most important predictor for bone mineral mass accrual during prepubertal growth [5]; and Madsen et al. [25] found LT to be a better predictor of BMD in eumenorrheic athletes (mean age+SD=20.8+2.5 yrs).Current research indicated that LT was the best predictor body composition component in both the adolescent and young adult groups (See Table 2)[26]. However, other research results are contrary to these findings. Reid et al. [27] observed BF was a better predictor of BMD than LT in premenopausal women (mean age+SD $=33+8 \mathrm{yrs}$ ). Lanyon, et al. found body weight and BF influenced BMD by placing an additional mechanical load on the skeleton [3], and Felson, et al. [27] found that body weight and BMI accounted for a substantial proportion $(8.9 \%-19.8 \%)$ of the total variance in BMD in women. The varying findings of studies to the association between BMD and body composition components question whether an additional approach to the examination of BMD may provide a deeper understanding of the metabolic process of BMD development and addressing the increased health and quality-of-life risks of reduced BMD.

The long term effects of reduced BMD due to the effects of the lack of weight-bearing physical activity, long term amenorrhea, and menopause are dramatic and demand attention [28-30]. Drinkwater's research revealed long-term cessation of menstrual periods due to exercise associated amenorrhea can lead to irreversible bone loss [31]. These are health and quality-of-life issues that must be of concern to at-risk females [32].

Allometric scaling can provide the developmental growth rate of the independent variable of the subjects which could be very useful in comparison to other groups or to normalized values, and review of the individual allometric coefficients indicate increasing and decreasing BMD levels throughout the life cycle.

While LT was the best body composition component to use as an independent variable to predict BMD in the current study, its applicability as a predictor variable for BMD in samples of mixed gender remains unknown, especially in using allometric scaling techniques to explain or describe the process of BMD in terms of LT growth. Determination of allometric exponents during different 
periods of the life cycle along with the variability due to gender and ethnic differences will be key in establishing its applicability in future BMD research.

In conclusion, the identification of new ways to examine BMD through the use of allometric scaling of body composition components, may provide deeper insights into understanding the complex association between BMD, body composition, and physical activity. The results of the current study reinforce the need for further investigation between BMD and allometric scaling of body composition components, especially in larger samples of mixed gender in different age groups.

\section{References}

1. Pocock NA, Eisman JA, Hopper JL, Yeates MG, Sambrook PN, et al. (1987) Genetic determinants of bone mass in adults: a twin study. J Clin Invest 80(3): 706-710.

2. Henderson S, Graham H, Mollan R, Riddoch C, Sheridan B, et al. (1989) Calcium homeostasis and exercise. Int Orthop 13(1): 69-73.

3. Lanyon L, Goodship A, Pye C, MacFie J (1982) Mechanically adaptive bone remodeling. J Biomech 15(3): 141-154.

4. Conroy BP, Earle RW (1994) Bone, muscle and connective tissue adaptations to physical activity. Essentials of Strength Training and Conditioning. Human Kinetics, Champaign 51- 66.

5. Vicente Rodríguez G (2006) How does Exercise Affect Bone Development during Growth? Sports Med 36(7): 561-569.

6. (1997) American College of Sports Medicine position stand on the female athlete triad, Medicine and Science in Sports and Exercise 29: i-ix.

7. Nattiv A, Loucks AB, Manore MM, Sanborn CF, Borgen JS, et al. (2007) The female athlete triad. Med Sci Sports Exerc 39(10): 1867-1882.

8. Gabel KA (2006) Special nutrition concerns for the female athlete. Curr Sports Med Rep 5(4): 187-191.

9. Wolinsky I (1994) Nutrition in Exercise and Sport. CRC Press, Boca Raton 508

10. Loucks AB, Horvath SM (1985) Athletic amenorrhea: a review. Med Sci Sports Exerc 17(1): 56-72.

11. Thein Nissenbaum JM, Rauh MJ, Carr KE, Loud KJ, McGuine TA (2011) Associations between disordered eating, menstrual dysfunction, and musculoskeletal injury among high school athletes. J Orthop Sports Phys Ther 41(2): 60-69.

12. Thein Nissenbaum JM, Carr KE (2011) Female athlete triad syndrome in the high school athlete. Phys Ther Sport 12(3): 108-116

13. Bonis M, Loftin M, Sothern M (2017) Using the lean tissue index as predictor variable for bone mineral density in elite, adolescent female cross-country runners. Journal of Sport and Human Performance 5(1): $1-9$.
14. Lohman TG, Ring K, Schmitz KH, Treuth MS, Loftin M, et al. (2006) Associations of body size and composition with physical activity in adolescent girls. Med Sci Sports Exerc 38(6): 1175-1181.

15. Shingleton A (2010) Allometry: The study of biological scaling. Education Knowledge 3(10): 2.

16. White J, Gould S (1965) Interpretation of the coefficient in the allometric equation. The American Naturalist 99(904): 5-18.

17. Huxley J, Tessier G (1936) Terminology of relative growth. Nature 137: 780-781.

18. Tartaruga M, De Medeiros M, Alberton C, Cadore E, Peyré Tartaruga LA, et al. (2010) Application of the allometric scalefor the submaximal oxygen uptake in runners and rowers. Biol Sport 27(4): 297-300.

19. Loftin M, Sothern M, Trosclair L, O Hanlon A, Miller J, et al. (2001) Scaling VO(2) peak in obese and non-obese girls. Obes Res 9(5): 290-296.

20. Nichols JF, Rauh MJ, Lawson MJ, Ji M, Barkai HS (2006) Prevalence of the female athlete triad syndrome among high school athletes. Arch Pediatr Adolesc Med 160(2): 137-142.

21. Nattiv A, Lynch L (1994) The female athlete triad: managing an acute risk to long term health. The Physician and Sportmedicine 22(1): 6068.

22. Huxley J (1924) Constant differential growth-ratios and their significance. Nature 114: 895-896.

23. West G, Brown J, Enquist B (1997) A general model for the origin of allometrical scaling laws in biology. Science 276(5309): 122-126.

24. Klieber M (1932) Body size and metabolism. Hilgardia 6(11): 315-351.

25. Madsen K, Adams W, Van Loan M (1998) Effects of physical activity, body weight and composition, and muscular strength on bone density in young women. Med Sci Sports Exerc 30(1): 114-120.

26. Reid I, Plank L, Evans M (1992) Fat mass is an important determinant of whole body bone mineral density in premenopausal women but not in men. Journal of Clinical Endocrinology and Metabolism 75(3): 779-782.

27. Felson D, Zhang Y, Hannan M, Anderson J (1993) Effects of weight and body mass index on bone mineral density in men and women: The Framingham Study. J Bone Miner Res 8(5): 567-573.

28. Dhuper S, Warren M, Brooks Gunn J, Fox R (1990) Effects of hormonal status on bone density in adolescent girls. Journal of Clinical Endocrinology and Metabolism 71 (5): 1083-1088.

29. Gutin B, Kasper M (1992) Can vigorous exercise play a role in osteoporosis prevention. Osteoporosis Int 2(2): 55-69.

30. Zanker CL, Swaine IL (1998) Relation between bone turnover, osteodiol, and energy balance in women distance runners. Br J Sports Med 32(2): 167-171.

31. Drinkwater BL, Nilson K, Bremner WI, Southworth MB, Shainholtz $S$ (1984) Bone mineral content of amenorrheic and eumenorrheic athletes. N Engl J Med 311(5): 277-281.

32. Waldrop J (2005) Early identification and interventions for female athlete triad. J Pediat Health Care 19(4): 213-220. 